\title{
Image-Based Tracking of Anticancer Drug-Loaded Nanoengineered Polyelectrolyte Capsules in Cellular Environments Using a Fast Benchtop Mid-Infrared (MIR) Microscope
}

Rabah Mouras, ${ }^{\dagger}$ Mohamed R. Noor, ${ }^{\ddagger}$ Laura Pastorino, ${ }^{\S}$ Enrico Bagnoli, ${ }^{\ddagger},, \#$ Aladin Mani, ${ }^{\dagger}$ Edel Durack, ${ }^{\ddagger}$ Alexei Antipov, ${ }^{\|}$Francesca D’Autilia, ${ }^{\perp}$ Paolo Bianchini, ${ }^{\perp}$ () Alberto Diaspro, ${ }^{\perp}$ Tewfik Soulimane, ${ }^{*}$ Christophe Silien, ${ }^{\dagger}$ Carmelina Ruggiero, ${ }^{\S}$ and Syed A. M. Tofail ${ }^{*}{ }^{\dagger}$

\footnotetext{
${ }^{\dagger}$ Department of Physics, Bernal Institute and ${ }^{\star}$ Department of Chemical Sciences, Bernal Institute, University of Limerick, Castletroy, Limerick V94 T9PX, Ireland

${ }^{\S}$ Department of Informatics, Bioengineering, Robotics and Systems Engineering, University of Genova, Via Opera Pia, 13, 16145 Genova, Italy

"PlasmaChem GmbH, Schwarzschildstr. 10, 12489 Berlin, Germany

${ }^{\perp}$ Nanophysics, Istituto Italiano di Tecnologia, Via Morego, 30, 16163 Genova, Italy
}

\section{Supporting Information}

\begin{abstract}
Drug delivery monitoring and tracking in the human body are two of the biggest challenges in targeted therapy to be addressed by nanomedicine. The ability of imaging drugs and micro-/nanoengineered drug carriers and of visualizing their interactions at the cellular interface in a labelfree manner is crucial in providing the ability of tracking their cellular pathways and will help understand their biological impact, allowing thus to improve the therapeutic efficacy. We present a fast, label-free technique to achieve high-resolution imaging at the mid-infrared (MIR) spectrum that provides chemical information. Using our custom-made benchtop

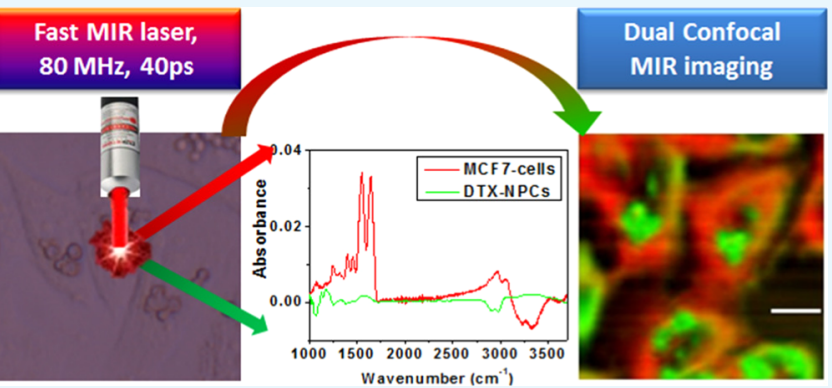
infrared microscope using a high-repetition-rate pulsed laser $(80 \mathrm{MHz}, 40 \mathrm{ps})$, we were able to acquire images with subwavelength resolution $(0.8 \times \lambda)$ at very high speeds. As a proof-of-concept, we embarked on the investigation of nanoengineered polyelectrolyte capsules (NPCs) containing the anticancer drug, docetaxel. These NPCs were synthesized using a layer-by-layer approach built upon a calcium carbonate $\left(\mathrm{CaCO}_{3}\right)$ core, which was then removed away with ethylenediaminetetraacetic acid. The obtained MIR images show that NPCs are attached to the cell membrane, which is a good step toward an efficient drug delivery. This has been confirmed by both three-dimensional confocal fluorescence and stimulated emission depletion microscopy. Coupled with additional instrumentation and data processing advancements, this setup is capable of video-rate imaging speeds and will be significantly complementing current super-resolution microscopy techniques while providing an unperturbed view into living cells.
\end{abstract}

\section{INTRODUCTION}

Optical spectroscopy techniques such as near-infrared (NIR), Raman, and Fourier Transform Infrared (FTIR) can potentially revolutionize disease diagnosis and therapy as an alternative or adjunct tool providing early diagnosis, surgical boundary identification, and therapeutic response monitoring. ${ }^{1-4}$ Novel treatment strategies involving personalized medicine and nanomedicine approach can be effectively monitored using these techniques. In particular, the convergence of pharmaceutical technology with nanotechnology in the field of nanomedicine offers a wealth of nanoparticles for therapeutic drug delivery ${ }^{5}$ that can overcome biological barriers. Imaging the interface between biology and the nonbiological delivery system is important in providing, for example, the ability of tracking cellular pathways of drugs and their carriers (micro- and nanoparticles), monitoring therapeutic responses, especially with respect to the biological building blocks, predicting antimicrobial or antibiofilm performances, ${ }^{6}$ and finding means to overcome specific delivery problems in vitro and in vivo. For example, recent studies show that only $0.7 \%$ injected dose of nanoparticles actually reach the targeted solid tumor in vivo.? The bioavailability of many hydrophobic cancer drugs is also limited and restricts efficient treatment.

Understanding the interaction of drugs and/or drug-loaded carriers with cellular membrane and subcellular components can unveil the entry route as well as the subsequent localization

Received: November 27, 2017

Accepted: April 17, 2018

Published: June 6, 2018 
of therapeutic agents within the cells. This can, in turn, provide important information regarding improvement in the design of drugs, carriers, and internal/external stimuli to improve therapeutic outcome. This had motivated researchers to develop techniques that can image biological specimens and track drug molecules at subcellular levels (say below $50 \mathrm{~nm}$ length scales). Among these techniques, fluorescence microscopy has traditionally played a key role in biointerface imaging due to the excellent specificity and contrast that can be achieved. Furthermore, technological advances in optical instrumentation, coupled with the development of chemistryspecific fluorescent probes, have allowed for an explosion of methods to study live cells at the micro- and nanoscales (e.g., confocal, stimulated emission depletion (STED), stochastic optical reconstruction microscopy (STORM), photo activated localization microscopy (PALM), structured illumination microscopy (SIM)). ${ }^{8-10}$ Fluorescence-based methods, however, suffer from a major drawback, which is the absolute requirement for labeling the features of interest, by using either fluorescent-labeled antibodies against specific proteins within the cell or probes that exhibit fluorescence upon binding to certain molecules. ${ }^{11} \mathrm{~A}$ direct consequence of labeling is the introduction of artifacts that can possibly alter the physiology of cells under investigation and are not able to reveal direct biochemical processes in real time due to photobleaching. The utilization of fluorophores is not always possible; hence, there is a need for the development of label-free techniques that can be used complementarily with fluorescence microscopy and are capable of real-time chemical and structural imaging under ambient conditions and high resolution.

Spontaneous Raman microscopy is a noninvasive technique that probes the intrinsic vibrational signatures of molecules, allows label-free imaging, circumvents the need for fluorescent or other extrinsic tags, and permits the visualization of the distribution of specific molecules with high sensitivity and specificity. ${ }^{12}$ Unfortunately, despite the efforts conducted to develop Raman microscopy, low signal levels limit this technique; hence, either a large number of molecules or long acquisition times are required, presenting significant limitations to the development of real-time studies.

Over the last 15 years, microscopy approaches using coherent Raman scattering (CRS) as a contrast mechanism had emerged as powerful techniques to address these limitations. Coherent Raman imaging, including stimulated Raman scattering (SRS) and coherent anti-Stokes Raman scattering (CARS), allows the enhancement of the weak Raman signal by means of nonlinear excitation 5 orders of magnitude over traditional Raman scattering, ${ }^{13}$ enabling the speeding up of the imaging speeds to video rates. ${ }^{14,15}$ However, the small penetration depths due to the use of the NIR lasers, the optical photodamage due to the two-photon absorption process, and the poor detection limit (millimolar to micromolar) have limited the application of CRS techniques mostly to in vitro applications. $^{16}$

Mid-infrared (MIR) microscopy is a powerful label-free technique that derives its contrast from the intrinsic absorption of the biomolecules at their vibrational fingerprint range and can provide a technical solution to these limitations. ${ }^{17-19}$ However, MIR instrumentation stagnated due mostly to the trade-off between spectral-spatial resolution and acquisition times. This has motivated researchers to look for alternative IR sources. To overcome the low brightness of Globar (thermal) sources, synchrotron sources (SRs) were used for FTIR microspectroscopy. The high brightness and stability of broadband SRs made them excellent for MIR imaging, and their coupling with focal plane array (FPA) detectors has greatly expanded the capability of infrared microscopes in terms of high rates of spectral acquisition at high signal-to-noise ratios. The use of multiple SR beams in a wide-field detection scheme allowed one to acquire diffraction-limited images at high spatial resolution and high speed, which has considerably extended the potential of infrared microscopy. ${ }^{20,21}$ However, the limited access and the high cost associated with the use of SRs restricted their use and made them unpractical.

Recently, new sources like quantum cascade lasers (QCLs) have emerged as an alternative to thermal or synchrotron sources, $^{22-24}$ and they are rapidly becoming practical midinfrared sources for a variety of applications, such as tracechemical sensing and health monitoring. However, their small tunability ranges (few micrometers) limit their use to exciting one or two vibrational modes. Thus, a good number of QCLs (half a dozen) are needed to exploit the whole fingerprint spectrum of biological specimens that span from 500 to 4000 $\mathrm{cm}^{-1}$, corresponding to a range between 2.7 and $16.7 \mu \mathrm{m}$. By assuming a linear scaling factor, then the acquisition time will be increased by another order of magnitude. ${ }^{24}$ One way to overcome the tunability issue is by exploiting the advantages of nonlinear optics to generate MIR laser beams that cover the whole fingerprint spectrum.

Benchtop MIR microscope is still diffraction limited, with a resolution that at best equals the used wavelength $(\lambda){ }^{25}$ whereas the use of synchrotron radiation with modifications can extend this limit to $1 / 2 \lambda$, corresponding to a pixel size of $0.54 \mu \mathrm{m} \times 0.54 \mu \mathrm{m} .{ }^{20}$ For instance, it takes $2-4 \mathrm{~h}$ to acquire an area of only $30 \mu \mathrm{m} \times 30 \mu \mathrm{m}$ as a fully diffraction-limited image using a synchrotron source ${ }^{26}$ equipped with a conventional confocal system. Longer acquisition times imposed by the MIR sources, in most practical cases, lead experimenters to choose larger aperture and step sizes, thereby compromising the achievable spatial resolution. ${ }^{24}$ Thus, the development of powerful, fast, and tunable MIR laser sources for microscopy will push forward IR imaging as a viable tool for disease diagnostics and medical research and provide real-time chemical and structural imaging under ambient conditions. ${ }^{27}$ Furthermore, the implementation of a confocal microscope will offer high-resolution imaging and potential three-dimensional (3D) imaging.

In this work, we present a high-speed, benchtop confocal MIR microscope capable of providing label-free chemical imaging at subwavelength spatial resolution. Our instrument is based on a high brightness compact fiber laser source with a repetition rate of $80 \mathrm{MHz}$ and 40 ps pulse duration. A state-ofthe-art fiber laser is used to pump a synchronously pumped optical parametric oscillator (SP-OPO) built around a fan-out MgO:PPLN crystal generating a signal and idlers beams varying from 1.5 to $2 \mu \mathrm{m}$ and from 2 to $4.8 \mu \mathrm{m}$, respectively. The SPOPO outputs are synchronously mixed inside a nonlinear crystal in a difference frequency generation (DFG) stage to generate a tunable mid-IR beam. The tunability of this type of lasers in the mid-IR range depends on the type of the nonlinear DFG crystal. Thus, our laser is tunable up to $8 \mu \mathrm{m}$ using a $\mathrm{CdSe}$ crystal and has the capability of extending its tunability up to $20 \mu \mathrm{m}$ using a $\mathrm{AgGaS}_{2}$ crystal. The ability of tuning the laser source throughout the entire MIR range paves the way that allows for a multiwavelength imaging of biological samples, akin to multichannel imaging in fluorescence microscopy. Such a 

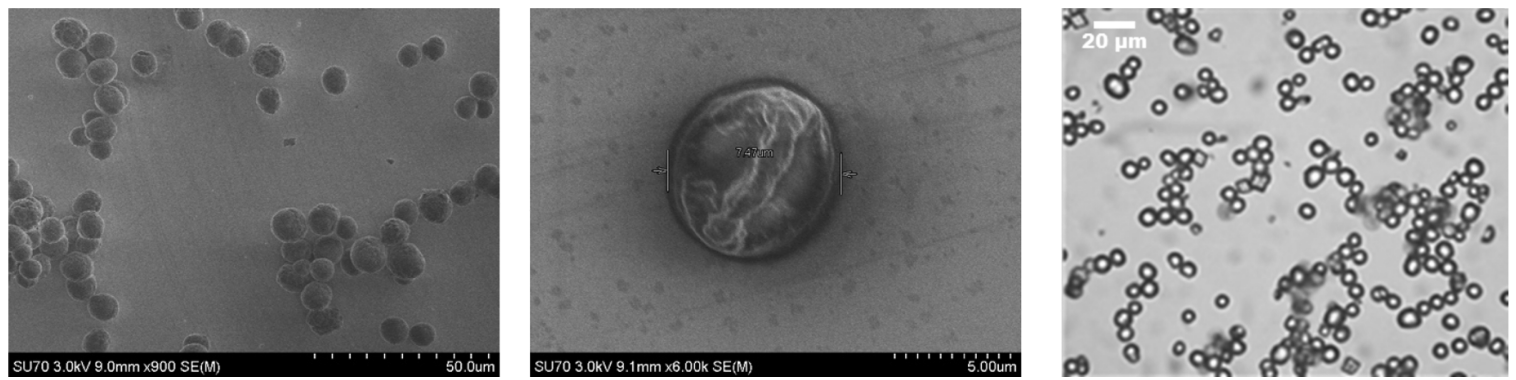

Figure 1. (Left) SEM images of $\mathrm{CaCO}_{3}$ cores used for the creation of PSS/PAH NPCs and (middle) (PSS/PAH) ${ }_{4}$ NPCs after the removal of $\mathrm{CaCO}_{3}$ core.The NPCs collapsed after the sample was dried. (Right) Corresponding wide-field optical image of PSS/PAH NPCs, which display a typical size distribution of about $1-6 \mu \mathrm{m}$. Scale bar $20 \mu \mathrm{m}$.

development has an immense potential for therapeutic monitoring and in vitro/in vivo diagnosis, including breast cancer, for which an early detection and treatment with constant monitoring are required.

As a proof-of-concept, we first performed in vitro imaging of docetaxel (DTX)-loaded NPCs composed of synthetic polyelectrolytes. $^{28-30}$ Namely, the polyanion, poly(styrene sulfonate) (PSS), and the polycation, poly(allylamine hydrochloride) (PAH), were used for the fabrication of a nanoengineered multilayered shell onto the surface of sacrificial calcium carbonate $\left(\mathrm{CaCO}_{3}\right)$ microparticles loaded with the chemotherapeutic drug, docetaxel. ${ }^{31}$

NPCs are seen as very promising for a broad range of applications in nanomedicine, such as drug delivery and biosensing, due to their tunability and easy fabrication. ${ }^{32-34}$ In this respect, the possibility of their tracking and visualization in a label-free manner could be useful to understand their interaction with cells and with intracellular organelles, ${ }^{35}$ in the view of perspective clinical applications. Recently, it has been demonstrated that NPCs can be functionalized with molecules as large as a membrane protein complex (ba3-cytochrome $c$ oxidase; $\sim 90 \mathrm{kDa}$ ) without perturbing their function. ${ }^{36}$ Herein, we characterized the in vitro interaction of such NPCs, loaded with docetaxel, with cancer cells by combining confocal and super-resolution (STED) fluorescence microscopies. These two techniques were used to validate the permeability variation of the NPCs by mainly looking at the entrance of the dye molecules (rhodamine) from the environmental solution into the NPC volume. ${ }^{36}$

In summary, data presented in this paper show the capabilities of our custom-made MIR microscope for chemical label-free imaging at high speeds and subwavelength resolution and high throughput imaging as well as its great potential in biomedical sciences. By adapting more appropriate instrumentation (fast lock-in amplifiers, electro-optical modulators), imaging at video rates will be possible. This will pave the way for real-time monitoring of drug delivery and for understanding its biological impact, which will support drug discovery and enhance the therapeutics efficacy.

\section{RESULTS AND DISCUSSION}

Synthesis of (PSS/PAH) NPCs. NPCs represent an excellent carrier for the encapsulation of drugs. ${ }^{37}$ In this study, NPCs loaded with DTX were fabricated by the alternate deposition of PSS and PAH onto the surface of docetaxelpreloaded $\mathrm{CaCO}_{3}$ microparticles. A range of microscopic techniques was applied to ensure the integrity of functionalized NPCs. Figure 1 shows the scanning electron microscopy
(SEM) and optical images of $\mathrm{CaCO}_{3}$ cores as well as the obtained (PSS/PAH) $)_{4}$ hollow NPCs. The SEM images show clearly that the NPCs collapsed after the sample was dried. Further coating of the NPCs with different moieties allows the NPCs to find more applications in biosciences and provides important properties to the NPCs such as (a) prolonged and sustained release (polymers), (b) magnetic nanoparticles (targeting, hyperthermia), (c) fluorescent nanoparticles and dyes (visualization), and others.

FTIR Spectroscopy. To facilitate the identification and differential imaging of empty and DTX-loaded NPCs, we acquired their FTIR absorption spectra to ensure that at least some spectral differences were present due to the presence of DTX. Both empty and DTX-loaded NPCs were kept in their solution state, and their FTIR spectra in the range between 1000 and $4000 \mathrm{~cm}^{-1}$ were acquired in attenuated total reflection (ATR) configuration. As shown in Figure 2, a

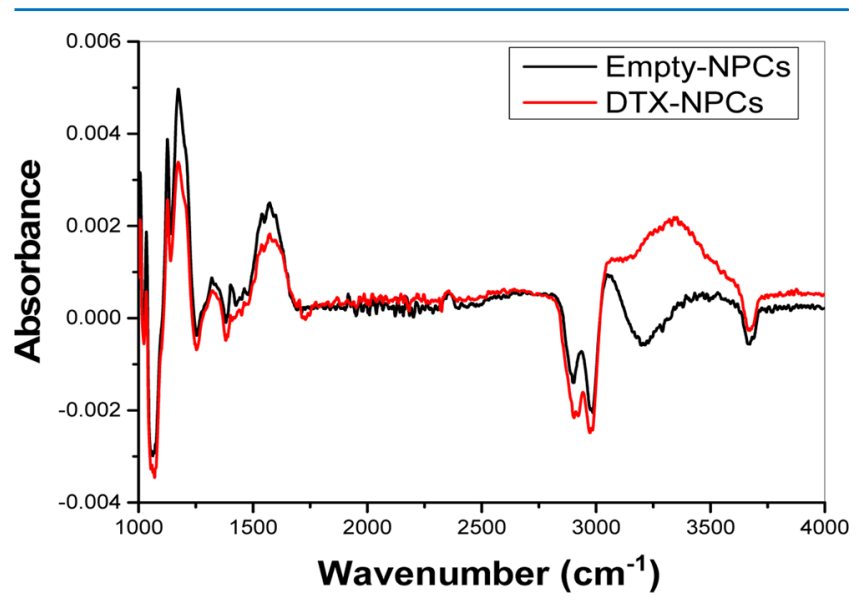

Figure 2. FTIR spectra of empty and DTX-loaded NPCs acquired in the ATR configuration in the range $1000-4000 \mathrm{~cm}^{-1}$ with $0.5 \mathrm{~cm}^{-1}$ step.

significant difference was apparent in the region of 3000-3500 $\mathrm{cm}^{-1}(3.3-2.9 \mu \mathrm{m})$, indicating the successful encapsulation of DTX in NPCs. We can see two distinct bands at 3200 and 3350 $\mathrm{cm}^{-1}$ that can be used to image both types of NPCs by tuning our MIR pulsed laser to excite these vibrational bands.

Fast, High-Resolution Label-Free MIR Imaging. Imaging of NPCS. We initially acquired the images of a calibration sample (silver thin film with microsized holes deposited on a microscope slide) to calibrate our setup and to determine the effective parameters and capabilities of our instrument such as imaging speed, resolution, and tunability. The obtained data 

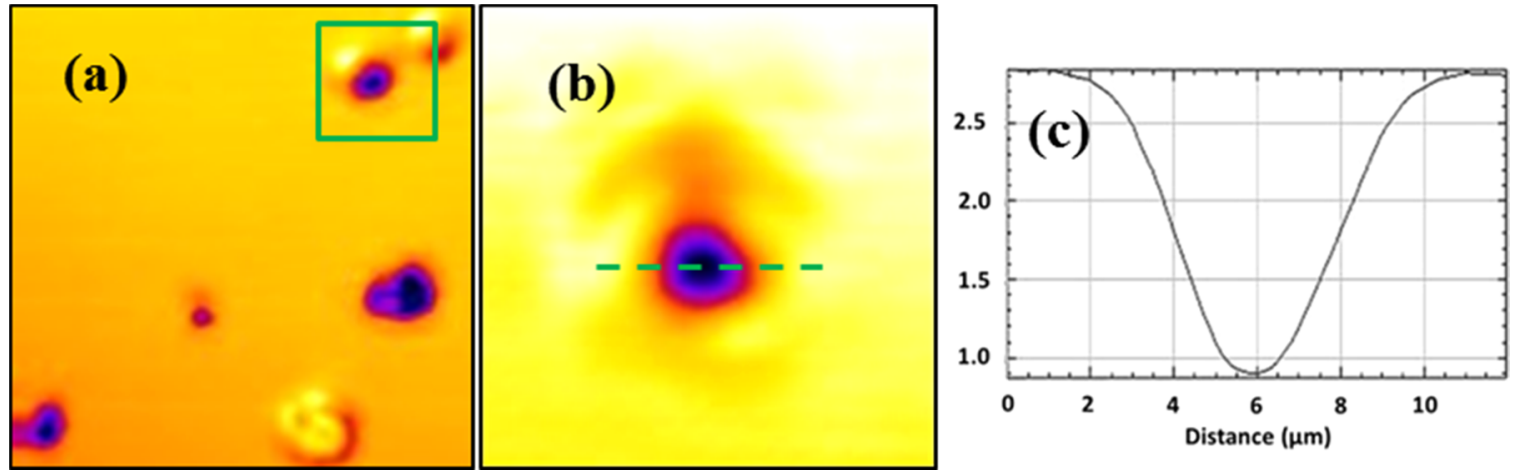

Figure 3. MIR image imaging of empty (PSS/PAH) ${ }_{4}$ NPCs. (a) MIR image taken at a wavelength of $3.125 \mu \mathrm{m}$ corresponding to a vibration at 3200 $\mathrm{cm}^{-1}$, image size $50 \mu \mathrm{m} \times 50 \mu \mathrm{m}$ and (b) $20 \mu \mathrm{m} \times 20 \mu \mathrm{m}$ zoom corresponding to the green square. The corresponding line profile (c) shows the size of the capsule to be $4 \mu \mathrm{m}$. All images are $256 \times 256$ pixels and have been acquired at $2 \mathrm{~ms}$ pixel/dwell time.

show the resolution of our microscope to be $0.8 \lambda$, indicating the subwavelength resolution imaging capability of our microscope (Figures S1 and S2). Then, empty PSS/PAH NPCs were dispersed on $\mathrm{CaF}_{2}$ coverslips and imaged using our MIR confocal microscope in transmission mode. $\mathrm{CaF}_{2}$ coverslips were used as they are transparent in the MIR region. The obtained MIR images are shown in Figure 3. The nonspherical shape indicates that the NPCs collapsed after the sample was dried, which is in good agreement with SEM data. The full width at half maximum of the profile along the capsule is used to determine the size of the NPCs.

MIR Imaging of Fixed Cells Incubated with DTX-Loaded NPCs and Dual-Channel Imaging. After demonstrating the proof-of-concept and capabilities of our microscope on a calibration sample and NPCs (Figures S1 and S2), this was then extended to an in vitro imaging of MCF7 cells incubated with 1 $\mathrm{mg} / \mathrm{mL}$ DTX-loaded NPCs for $48 \mathrm{~h}$ and then fixed in formaldehyde. To image the MCF cells, we tuned our laser to $3.5 \mu \mathrm{m}$ to excite the lipid stretch at $2850 \mathrm{~cm}^{-1}$. A control sample was used for comparison. We can see clearly that the IR intensity is homogeneous throughout the control sample; however, the MCF7 cells incubated with DTX-loaded NPCs show the presence of more intense features closely attached to the cell membrane (Figure 4).

Because both NPCs and cells membranes have a high absorption peak around $2850 \mathrm{~cm}^{-1}$ as shown in the FTIR spectra, these features are potential aggregates of DTX-loaded
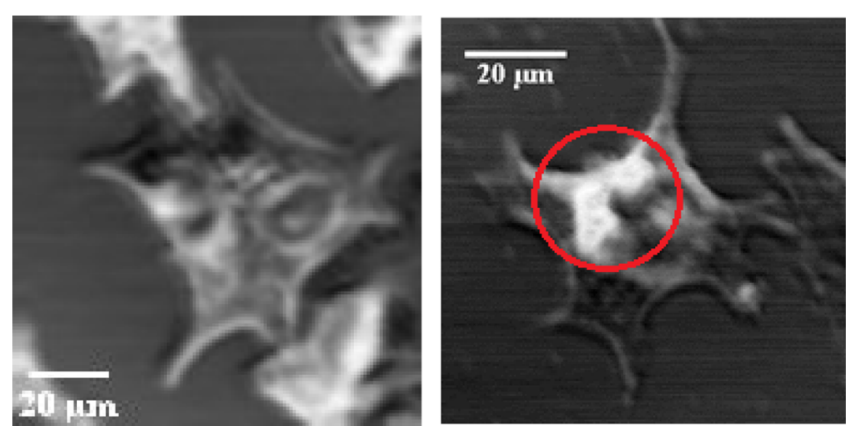

Figure 4. MIR image of MCF7 cells incubated with DTX-loaded NPCs. (Left) Control cells without any NPCs and (middle) cells incubated with $200 \mu \mathrm{L}$ of DTX-loaded NPCs at a concentration of 1 $\mathrm{mg} / \mathrm{mL}$ for $48 \mathrm{~h}$. The circle on the right (bright white spots) indicates potential aggregation of NPCs. Both images were obtained at 2850 $\mathrm{cm}^{-1}(\lambda=3.5 \mu \mathrm{m})$.
NPCs. Images are $256 \times 256$ pixels obtained at very high speed with a dwell time of $2 \mathrm{~ms} /$ pixel, resulting in a total acquisition time of $131 \mathrm{~s}$. We note that the imaging rate was limited by the acquisition parameters of the lock-in amplifier (time constant $=$ $1 \mathrm{~ms}$ ) and the beam modulation $(0.5 \mathrm{~Hz})$. Higher speeds with 1 $\mu$ s pixel dwell time are possible using fast lock-in amplifiers and electo-optical modulators, thus allowing imaging rates 3 orders of magnitude higher than the current speeds.

As our laser is monochromatic and only one wavelength can be used at the time, revealing the chemical contrast between different biological specimens can be achieved by wisely selecting two or three wavelengths and recording the images in sequence. Our laser features a mechanical tunability, allowing thus instantaneous change to the desired wavelength. This multiwavelength approach and fast tunability (Figure S2) represent an alternative solution to the slow broadband imaging and permit chemical imaging at different frequencies and will considerably accelerate the chemical imaging mode while conserving subwavelength resolution. To achieve this, we tuned our laser to two specific wavelengths of 3.4 and $2.95 \mu \mathrm{m}$ to image the cells and DTX-loaded NPCs, respectively. These wavelengths correspond to the $\mathrm{C}-\mathrm{H}\left(2940 \mathrm{~cm}^{-1}\right)$ and $\mathrm{N}-\mathrm{H}$ $\left(3400 \mathrm{~cm}^{-1}\right)$ bonds in cells and DTX-loaded NPCs, respectively. The obtained images are presented in Figure 5. The two images were overlaid to obtain a label-free dual-color image showing the attachment of the DTX-loaded NPCs (in green) to the cell membranes (in red). The obtained image is of the same quality as the confocal fluorescence images, yet without any labeling.

Our MIR microscope features a dual-analogue data acquisition interface (DAQ Physik Instrument), which allows us to record only two-dimensional images. Thus, we were not able to record 3D images as in confocal microscopy and to find out whether the NPCs were attached to the outer side of the cell membranes or have crossed the cell membrane. To validate our MIR findings, we used the more established confocal and STED microscopies.

Confocal and STED Microscopy of Living Cells. To assess the effect of empty and DTX-loaded NPCs on living cells, we embarked on a series of more established confocal and STED microscopies. As both techniques require exogenous labeling, and to visualize both cells and NPCs, we have consequently chosen two fluorescent probes with distinct peak excitation/ emission wavelength pairs (i.e., emission of one probe not overlapping with the excitation of another). Thus, cells were stained with Hoechst 33342 (Invitrogen, Paisley, U.K.), a dye 

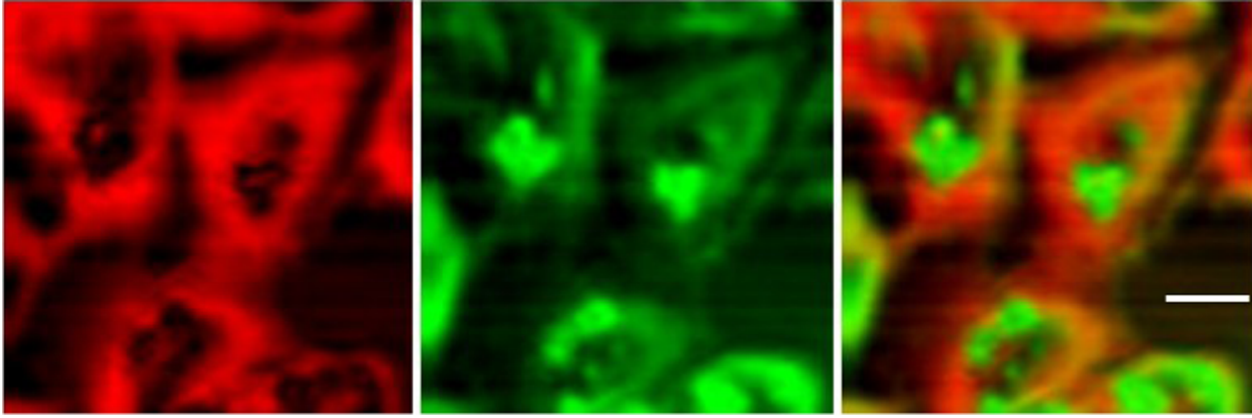

Figure 5. Dual-color high-resolution imaging of MCF7 breast cancer cell line incubated with DTX-loaded NPCs. The MIR laser was tuned to 3.4 $\mu \mathrm{m}$ (C-H bond) to image cells (red) and to $2.7 \mu \mathrm{m}(\mathrm{N}-\mathrm{H}$ bond) to image NPCs (green). The overlay of the two images (right) shows the attachment of the DTX-loaded NPCs to the cell membranes. Image size is $256 \times 256$ pixels acquired with 2 ms pixel dwell time, giving a total acquisition time of $131 \mathrm{~s}$. Scale bar $20 \mu \mathrm{m}$.

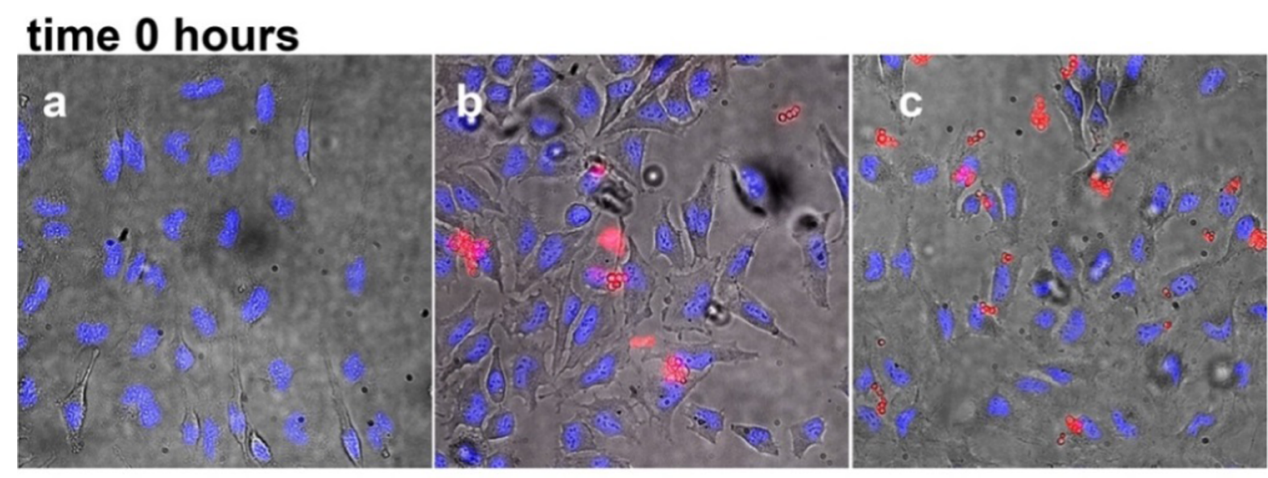

\section{time 2 hours}

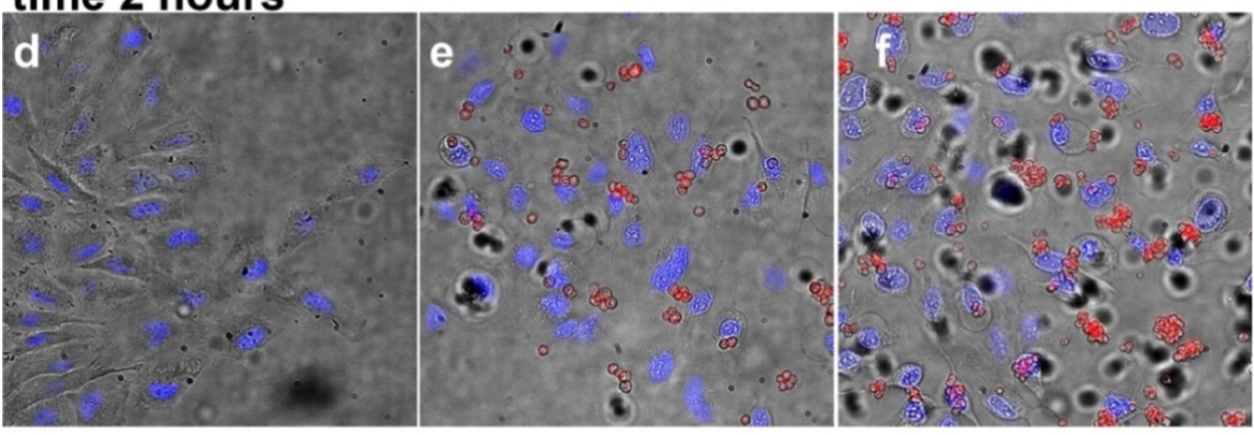

\section{time 4 hours}

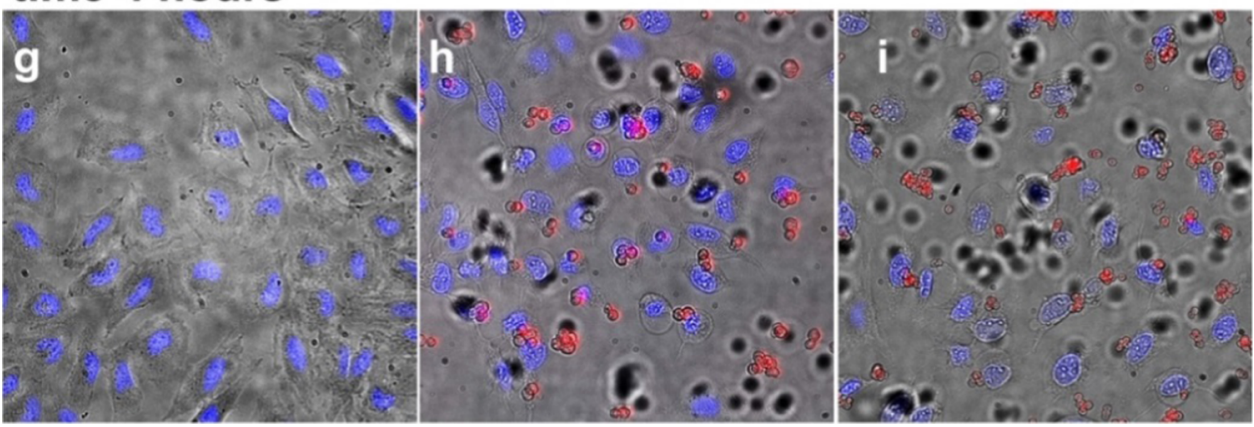

Figure 6. Wide-field fluorescence and transmission time-lapsed imaging of living HeLa cells in culture at different time points. Control cells without NPCs are shown in the panels (a), (d), (g) whereby cells behave normally, whereas those with empty and drug-loaded NPCs exhibit cytotoxic effects (panels $(b, e, h)$ and (c, f, i), respectively). The gray images were acquired in the transmission mode. The cell nuclei and NPCs were labeled with Hoechst dye targeted against DNA (blue) and Atto 590 (red), respectively.

emitting around $450 \mathrm{~nm}$, to visualize the nuclei, whereas the four layers of PAH/PSS NPCs were labeled using covalently functionalized PAH-ATTO 590 at the third layer. Atto 590
(ATTO-TEC GmbH, Siegen, Germany) is a dye that has its absorption peak at $593 \mathrm{~nm}$ and an emission peak at $622 \mathrm{~nm}$. Figure 6 shows time-lapsed imaging taken at 0,2 , and $4 \mathrm{~h}$ after 

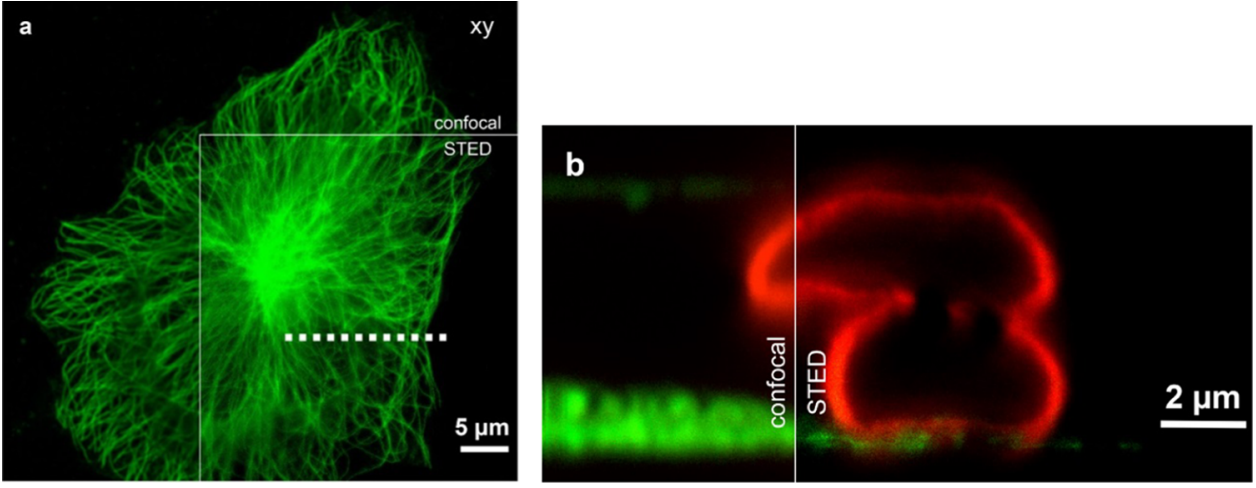

Figure 7. Multicolor 3D-STED images of HeLa cells treated with DTX-loaded NPCs. In panel (a), the cells were imaged as a single $x y$ section with microtubules labeled with ATTO $647 \mathrm{~N}$-labeled antibody. The corresponding (dotted line) $x z$ section is shown in panel (b), where the two ATTO 590-labeled NPCs are visible.

incubation with empty and DTX-loaded NPCs. A control sample was used to assess the cytotoxicity of the NPCs. The obtained images show that even after $4 \mathrm{~h}$, the control sample shows healthy cells (Figure 6, panels a, d, and g); however, cells incubated with empty NPCs start to die after $2 \mathrm{~h}$, and almost all of the cells were dead after $4 \mathrm{~h}$. We observed that the toxicity effect suggested by the 3-(4,5-dimethylthiazol-2-yl)-2,5-diphenyltetrazolium bromide (MTT) assay (Figure S3) is confirmed microscopically, where high concentrations of (PSS/PAH) NPCs have a toxic effect and already exhibit some cell killing properties (Figure 6, panels b, e, and h). Furthermore, the presence of DTX accelerated cell death, indicating that the functionalization of NPCs does not alter the properties of DTX (Figure 6, panels c, f, and i). This finding suggests that these hollow NPCs can be used as therapeutics carriers, and further functionalization of their shell will make them very promising for targeted therapy.

Then, the shape and morphology of the NPCs when exposed onto the cells were checked by both $3 \mathrm{D}$ confocal and STED microscopies, as shown in Figure 7. We can see clearly that the NPCs attach to the cell membrane, which is a good step toward DTX delivery, by forcing the rupture of the capsule's shell, allowing thus an efficient delivery of DTX to the cells. The NPCs do not adopt a position optimized enough for a timed, targeted release of correct dosage. More work needs to be performed to optimize the delivery process by controlling the opening of the NPCs as well as their attachment to the cell membrane. In an ideal case, it would be preferable for an individual capsule to contact a single cell or at least reproducibly if the ratio is not $1: 1$. The optical and SEM images of the capsule already indicate that a high monodispersity could not be achieved within our experimental condition. Together with advanced drug release modeling and pharmacokinetics, we anticipate these parameters to be further optimized.

These data are only the beginning of a larger series of investigation into label-free biological samples that can be exploited by our instrument. As our view of biology has thus far been mostly shaped by fluorescence-based microscopy techniques, label-free super-resolution MIR microscopies herald a new era whereby commercial probe availability will no longer restrict interesting studies. We expect MIR microscopy to be initially subject to complementary imaging for cross-validation and multimodality-based understanding into unique sample environments.

\section{CONCLUSIONS}

We have developed a benchtop MIR microscope based on high repetition and fast tunable OPO laser. We used our custommade microscope to image MCF7 cancer cells incubated with PSS/PAH DTX-loaded NPCs in a label-free manner. The combination of MIR imaging with super-resolution methods proposed in this work allows for the investigation of drug carrier interaction with the cell membrane and the subsequent delivering of anticancer drugs. The ability of directly visualizing individual NPCs and cell organelles at subwavelength resolution and at high speeds without adding extraneous tags will yield crucial information on biochemical processes like the interaction of drug carriers (NPCs) with cells as well as intracellular delivery of drugs. Complementing MIR with superresolution microscopy will provide more information about the interaction of nanoparticles with cells, the intracellular trafficking of drugs, their localization within the cells, and their subsequent therapeutic effect. In this framework, the multimodality IR with super-resolution microscopy represents a powerful tool toward the in vitro monitoring of therapeutics delivery, as well as translating this work toward in vivo applications.

\section{MATERIALS AND METHODS}

Polyelectrolyte Capsule Synthesis and FTIR Spectroscopy. Cationic PAH $\left(M_{\mathrm{w}} 70 \mathrm{kDa}\right.$, Sigma-Aldrich) and anionic PSS ( $M_{\mathrm{w}} 70 \mathrm{kDa}$, Sigma-Aldrich) were used as synthetic polyelectrolytes for shell deposition. NPCs were fabricated following a well-established procedure. ${ }^{28-30,36}$ Specifically, four polyelectrolyte bilayers were deposited onto DTX-preloaded $(1 \% \mathrm{w} / \mathrm{v}) \mathrm{CaCO}_{3}$ microparticles $(6 \mu \mathrm{m}$ in diameter) (PlasmaChem $\mathrm{GmbH}$ ). Ethylenediaminetetraacetic acid (EDTA) (Sigma-Aldrich) was used as a complexing agent for the removal of the $\mathrm{CaCO}_{3}$ template. PSS and $\mathrm{PAH}$ were prepared in pure water at a concentration of $2 \mathrm{mg} / \mathrm{mL}, \mathrm{pH} 6.5$, and their adsorption time was $10 \mathrm{~min}$. Four bilayers were deposited onto the surface of the particles; after each deposition step, the dispersion of the covered particles was centrifuged ( $2500 \mathrm{rpm}$ for $5 \mathrm{~min}$ ) and the precipitated covered particles were separated from the solution. These particles were washed three times in pure water. The $\mathrm{CaCO}_{3}$ cores were then dissolved by their dispersion in $0.1 \mathrm{M}$ EDTA, $\mathrm{pH} 5$, followed by three washings in pure water and redispersed in $0.1 \mathrm{M}$ phosphate buffered solution (PBS) at $\mathrm{pH}$ 7.4. 
FTIR spectra of empty and DTX-loaded NPCs were acquired in the ATR configuration (Perkin-Elmer Spectrum 100) in the solution state without using the crystal, by averaging 100 individual spectra for each. The instrument was blanked with PBS. Both optical microscopy and scanning electron microscopy (SEM) were used to determine the size of individual particles and their integrity.

Cell Culture and Incubation with NPCs. MCF7 breast cancer cells and HeLa cervical cancer cells were cultured in Dulbecco's modified Eagle's medium with fetal bovine serum and $1 \%$ penicillin-streptomycin at $37{ }^{\circ} \mathrm{C}$ under a $5 \% \mathrm{CO}_{2}$ atmosphere. Before delivery, NPCs were sterilized through UVC irradiation $\left(\lambda_{\max }=254 \mathrm{~nm}\right)$ as installed in a biological safety cabinet for $2 \mathrm{~h}$. The integrity of NPCs before and after UV sterilization was checked using UV-visible spectroscopy. The obtained data showed that UV irradiation of NPCs did not induce any structural changes, which was also confirmed through confocal laser scanning microscopy of NPCs loaded with rhodamine. This observation is in contrast to previously reported shrinking of the polymeric shell. ${ }^{38}$ Then, we performed MTT (3-(4,5-dimethylthiazol-2-yl)-2,5-diphenyltetrazolium bromide) tetrazolium reduction assay ${ }^{39,40}$ - known for assessing cell metabolic activity and cytotoxicity-to determine their degree of cytotoxicity (see the Supporting Information, Figure S3a,b).

MIR Microscopy. For label-free MIR imaging, a custommade tabletop MIR confocal microscope was used. The microscope is equipped with a compact and high-repetitionrate $(80 \mathrm{MHz})$ picosecond $(30 \mathrm{ps})$ optical parametric oscillator (OPO) (Laserspec, Belgium) pumped with a picosecond fiber laser (40 ps, $1030 \mathrm{~nm}$ ) (Multitel Ltd, Belgium). The OPO is tunable from 2 to $8 \mu \mathrm{m}$, resulting in an observable spectral range of $1250-5000 \mathrm{~cm}^{-1}$, and also allows excitation/detection in both reflection and transmission modes. To acquire images, the sample is scanned using a Nano-cube XYZ scanner from PI (P-611.3 NanoCube XYZ Piezo Stage, Physical Instrument), which is controlled via E-664 Controller. The signal is detected using an mercury cadmium telluride (MCT) photoconductive detector (P-2748-42, Hamamatsu) through a lock-in amplifier, with the laser beam mechanically chopped at $500 \mathrm{~Hz}$. The data acquisition is performed via our own image acquisition software that allows image collection at the rate of $1 \mathrm{~ms} /$ pixel dwell time. Note here that this long pixel dwell time was imposed by the time constant of the lock-in amplifier used to collect and amplify the signal. For high-level signals, we achieved a $100 \mu \mathrm{s}$ pixel dwell time by surpassing the use of a lock-in amplifier. A calibration sample (Ag film calibration slide with $5 \mu \mathrm{m}$ holes and $500 \mathrm{~nm} \mathrm{Ag}$ nanoparticles) was used to align the microscope and to estimate the lateral resolution of the system.

Confocal and Super-Resolution Microscopy. Confocal laser scanning microscopy was performed to study the permeability variation looking mainly at the entrance of the dye molecules (rhodamine) from the environmental solution into the NPC volume. The images were obtained using a Leica TCS SP5 STED-CW (Leica Microsystems, Mannheim, Germany) inverted confocal laser scanning microscope equipped with a supercontinuum laser covering the visible spectrum in the range between 470 and $640 \mathrm{~nm}$. The images were collected using a Leica $1006 \mathrm{HCX}$ PL APO STEDorange NA 1.40 oil immersion objective (Leica Microsystems CMS, Mannheim, Germany) with an excitation at $561 \mathrm{~nm}$ and an emission between 570 and $620 \mathrm{~nm}$, with no lines averaging at a speed of $1 \mathrm{kHz}$ per line, a pixel dwell time of $2 \mu \mathrm{s}$, and a pinhole size of 0.8 Airy. Under this imaging configuration, typical confocal resolution is on the order of $200 \mathrm{~nm}$ in the lateral direction and $500 \mathrm{~nm}$ in the axial direction. The morphology of the NPCs in contact with cells was also studied with multicolor 3D-STED super-resolution nanoscopy.

\section{ASSOCIATED CONTENT}

\section{S Supporting Information}

The Supporting Information is available free of charge on the ACS Publications website at DOI: 10.1021/acsomega.7b01859.

Microscope calibration and subwavelength resolution; fast tunability and wavelength-dependent chemical imaging; cytotoxicity analysis and MTT assays (PDF)

\section{AUTHOR INFORMATION}

\section{Corresponding Author}

*E-mail: Tofail.Syed@ul.ie. Phone: +353 61234132.

ORCID

Rabah Mouras: 0000-0001-5100-4739

Paolo Bianchini: 0000-0001-6457-751X

Present Address

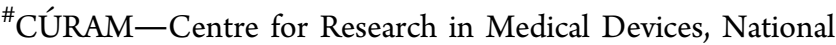
University of Ireland, Galway H91 CF50, Ireland (E.B.).

\section{Notes}

The authors declare no competing financial interest.

\section{ACKNOWLEDGMENTS}

The research leading to these results has received funding from the European Community's Seventh Framework Program, LANIR (FP7/20012-2015), under grant agreement no. 280804. R.M. acknowledges Enterprise Ireland for the funding under grant agreement no. IP20150404.This article reflects the views only of the authors, and the Commission cannot be held responsible for any use that may be made of the information contained therein.

\section{REFERENCES}

(1) Devpura, S.; Barton, K. N.; Brown, S. L.; Palyvoda, O.; Kalkanis, S.; Naik, V. M.; Siddiqui, F.; Naik, R.; Chetty, I. J. Vision 20/20: The role of Raman spectroscopy in early stage cancer detection and feasibility for application in radiation therapy response assessment. Med. Phys. 2014, 41, No. 050901.

(2) Evers, D. J.; Hendriks, B.; Lucassen, G.; Ruers, T. Optical spectroscopy: current advances and future applications in cancer diagnostics and therapy. Future Oncol. 2012, 8, 307-320.

(3) Spliethoff, J. W.; Evers, D. J.; Jaspers, J. E.; Hendriks, B. H. W.; Rottenberg, S.; Ruers, T. J. M. Monitoring of Tumor Response to Cisplatin Using Optical Spectroscopy. Transl. Oncol. 2014, 7, 230239.

(4) Kullaa, A. M.; Singh, S. P.; Mikkonen, J. W.; Koistinen, A. P. Optical spectroscopy in therapy response monitoring: an awakening giant. Spectrosc. Eur. 2015, 27, 6-10.

(5) Mirza, A. Z.; Siddiqui, F. A. Nanomedicine and drug delivery: a mini review. Int. Nano Lett. 2014, 4, No. 94.

(6) Koumoulos, E. P.; Tofail, S. A. M.; Silien, C.; De Felicis, D.; Moscatelli, R.; Dragatogiannis, D. A.; Bemporad, E.; Sebastiani, M.; Charitidis, C. A. Metrology and nano-mechanical tests for nanomanufacturing and nano-bio interface: Challenges \& future perspectives. Mater. Des. 2018, 137, 446-462.

(7) Wilhelm, S.; Tavares, A. J.; Dai, Q.; Ohta, S.; Audet, J.; Dvorak, H. F.; Chan, W. C. W. Analysis of nanoparticle delivery to tumours. Nat. Rev. Mater. 2016, 1, No. 16014. 
(8) Maglione, M.; Sigrist, S. J. Seeing the forest tree by tree: superresolution light microscopy meets the neurosciences. Nat. Neurosci. 2013, 16, 790 .

(9) Lippincott-Schwartz, J.; Manley, S. Putting super-resolution fluorescence microscopy to work. Nat. Methods 2009, 6, 21-23.

(10) Seeing in Super-Resolution. Nat. Methods 2014, 11, 1183. DOI: $10.1038 /$ nmeth.3206.

(11) Fernández-Suárez, M.; Ting, A. Y. Fluorescent probes for superresolution imaging in living cells. Nat. Rev. Mol. Cell Biol. 2008, 9, 929-943.

(12) Choo-Smith, L. P.; Edwards, H. G.; Endtz, H. P.; Kros, J. M.; Heule, F.; Barr, H.; Robinson, J. S., Jr.; Bruining, H. A.; Puppels, G. J. Medical applications of Raman spectroscopy: from proof of principle to clinical implementation. Biopolymers 2002, 67, 1-9.

(13) Mouras, R.; Bagnaninchi, P.; Downes, A.; Muratore, M.; Elfick, A. Non linear optical microscopy of adipose-derived stem cells induced towards osteoblasts and adipocytes. Proc. SPIE 2011, 8086, No. 80860 Q.

(14) Evans, C. L.; Potma, E. O.; Puoris'haag, M.; Côté, D.; Lin, C. P.; Xie, X. S. Chemical imaging of tissue in vivo with video-rate coherent anti-Stokes Raman scattering microscopy. Proc. Natl. Acad. Sci. U.S.A. 2005, 102, 16807.

(15) Camp, C. H., Jr.; Lee, Y. J.; Heddleston, J. M.; Hartshorn, C. M.; Walker, A. R. H.; Rich, J. N.; Lathia, J. D.; Cicerone, M. T. High-speed coherent Raman fingerprint imaging of biological tissues. Nat. Photonics 2014, 8, 627 .

(16) Arora, R.; Petrov, G. I.; Yakovlev, V. V. Analytical capabilities of coherent anti-Stokes Raman scattering microspectroscopy. J. Mod. Opt. 2008, 55, 3237-3254.

(17) Atkins, P. W.; De Paula, J. Elements of Physical Chemistry, 5th ed.; Oxford University Press: Oxford, 2009.

(18) Demtröder, W. Laser Spectroscopy: Basic Concepts and Instrumentation, 3rd ed.; Berlin: Springer-Verlag, 2003.

(19) Steele, D. Infrared Spectroscopy: Theory. In Handbook of Vibrational Spectroscopy; John Wiley \& Sons, Ltd, 2006.

(20) Nasse, M. J.; Walsh, M. J.; Mattson, E. C.; Reininger, R.; Kajdacsy-Balla, A.; Macias, V.; Bhargava, R.; Hirschmugl, C. J. Highresolution Fourier-transform infrared chemical imaging with multiple synchrotron beams. Nat. Methods 2011, 8, 413-416.

(21) Hirschmugl, C. J.; Gough, K. M. Fourier transform infrared spectrochemical imaging: review of design and applications with a focal plane array and multiple beam synchrotron radiation source. Appl. Spectrosc. 2012, 66, 475-491.

(22) Bassan, P.; Weida, M. J.; Rowlette, J.; Gardner, P. Large scale infrared imaging of tissue micro arrays (TMAs) using a tunable Quantum Cascade Laser (QCL) based microscope. Analyst 2014, 139, $3856-3859$.

(23) Kröger, N.; Egl, A.; Engel, M.; Gretz, N.; Haase, K.; Herpich, I.; Kranzlin, B.; Neudecker, S.; Pucci, A.; Schonhals, A.; Vogt, J.; Petrich, W. Quantum cascade laser-based hyperspectral imaging of biological tissue. J. Biomed. Opt. 2014, 19, No. 111607.

(24) Bird, B.; Baker, M. J. Quantum Cascade Lasers in Biomedical Infrared Imaging. Trends Biotechnol. 2015, 33, 557-558.

(25) Kumar, S.; Shabi, T. S.; Goormaghtigh, E. A FTIR Imaging Characterization of Fibroblasts Stimulated by Various Breast Cancer Cell Lines. PLoS One 2014, 9, No. e111137.

(26) Dumas, P.; Jamin, N.; Teillaud, J. L.; Miller, L. M.; Beccard, B. Imaging capabilities of synchrotron infrared microspectroscopy. Faraday Discuss. 2004, 126, 289-302 ; discussion 303-311..

(27) Dumas, P.; Sockalingum, G. D.; Sule-Suso, J. Adding synchrotron radiation to infrared microspectroscopy: what's new in biomedical applications? Trends Biotechnol. 2007, 25, 40-44.

(28) Sukhorukov, G. B.; Donath, E.; Lichtenfeld, H.; Knippel, E.; Knippel, M.; Budde, A.; Möhwald, H. Layer-by-layer self assembly of polyelectrolytes on colloidal particles. Colloids Surf., A 1998, 137, 253266.

(29) Volodkin, D. V.; Larionova, N. I.; Sukhorukov, G. B. Protein encapsulation via porous $\mathrm{CaCO} 3$ microparticles templating. Biomacromolecules 2004, 5, 1962-1972.
(30) Volodkin, D. V.; Petrov, A. I.; Prevot, M.; Sukhorukov, G. B. Matrix polyelectrolyte microcapsules: new system for macromolecule encapsulation. Langmuir 2004, 20, 3398-3406.

(31) Stanciu, S. G.; Tranca, D. E.; Ruggiero, C.; Stanciu, G. A.; Dellacasa, E.; Antipov, A.; Hristu, R.; Pastorino, L. Combined far-field, near-field and topographic imaging of nano-engineered polyelectrolyte capsules. Mater. Lett. 2016, 183, 105-108.

(32) De Geest, B. G.; De Koker, S.; Sukhorukov, G. B.; Kreft, O.; Parak, W. J.; Skirtach, A. G.; Demeester, J.; De Smedt, S. C.; Hennink, W. E. Polyelectrolyte microcapsules for biomedical applications. Soft Matter 2009, 5, 282-291.

(33) Zyuzin, M. V.; Diez, P.; Goldsmith, M.; Carregal-Romero, S.; Teodosio, C.; Rejman, J.; Feliu, N.; Escudero, A.; Almendral, M. J.; Linne, U.; Peer, D.; Fuentes, M.; Parak, W. J. Comprehensive and Systematic Analysis of the Immunocompatibility of Polyelectrolyte Capsules. Bioconjugate Chem. 2017, 28, 556-564.

(34) Xuan, M.; Zhao, J.; Shao, J.; Du, C.; Cui, W.; Duan, L.; Qi, W.; $\mathrm{Li}$, J. Recent progresses in layer-by-layer assembled biogenic capsules and their applications. J. Colloid Interface Sci. 2017, 487, 107-117.

(35) Rivera-Gil, P.; De Koker, S.; De Geest, B. G.; Parak, W. J. Intracellular processing of proteins mediated by biodegradable polyelectrolyte capsules. Nano Lett. 2009, 9, 4398-4402.

(36) Pastorino, L.; Dellacasa, E.; Noor, M. R.; Soulimane, T.; Bianchini, P.; D’Autilia, F.; Antipov, A.; Diaspro, A.; Tofail, S. A.; Ruggiero, C. Multilayered polyelectrolyte microcapsules: interaction with the enzyme cytochrome C oxidase. PLoS One 2014, 9, No. e112192.

(37) Parakhonskiy, B. V.; Yashchenok, A. M.; Möhwald, H.; Volodkin, D.; Skirtach, A. G. Release from Polyelectrolyte Multilayer Capsules in Solution and on Polymeric Surfaces. Adv. Mater. Interfaces 2017, 4, No. 1600273.

(38) Katagiri, K.; Matsuda, A.; Caruso, F. Effect of UV-Irradiation on Polyelectrolyte Multilayered Films and Hollow Capsules Prepared by Layer-by-Layer Assembly. Macromolecules 2006, 39, 8067-8074.

(39) Mosmann, T. Rapid colorimetric assay for cellular growth and survival: application to proliferation and cytotoxicity assays. J. Immunol. Methods 1983, 65, 55-63.

(40) Gerlier, D.; Thomasset, N. Use of MTT colorimetric assay to measure cell activation. J. Immunol. Methods 1986, 94, 57-63. 